\title{
Non-Ewald methods: theory and applications to molecular systems
}

\author{
Ikuo Fukuda $\cdot$ Haruki Nakamura
}

Received: 15 May 2012 / Accepted: 21 June 2012 / Published online: 2 August 2012

(C) The Author(s) 2012. This article is published with open access at Springerlink.com

\begin{abstract}
Several non-Ewald methods for calculating electrostatic interactions have recently been developed, such as the Wolf method, the reaction field method, the preaveraging method, and the zero-dipole summation method, for molecular dynamics simulations of various physical systems, including biomolecular systems. We review the theories of these approaches and their potential applications to molecular simulations, and discuss their relationships.
\end{abstract}

Keywords Molecular dynamics - Electrostatic interaction · Reaction field method · Pre-averaging method · Wolf method $\cdot$ Zero-dipole summation method

\section{Introduction}

Molecular simulation via molecular dynamics (MD) or Monte-Carlo calculations is a powerful tool for understanding the nature of biomolecular systems, including water, proteins, lipids, DNAs, and their complexes. In these simulations, appropriate treatment of the electrostatic interactions is critical, since they play essential roles in a number of systems, by maintaining physical structures, generating chemical properties, and performing biological functions (Patra et al. 2004; Koehl 2006; Reif et al. 2009; Srivastava et al. 2010).

Special issue: Computational Biophysics

I. Fukuda $(\triangle)$

RIKEN (The Institute of Physical and Chemical Research),

2-1 Hirosawa,

Wako, Saitama 351-0198, Japan

e-mail: ifukuda@riken.jp

H. Nakamura

Institute for Protein Research, Osaka University,

3-2 Yamadaoka,

Suita, Osaka 565-0871, Japan
Specifically, the Coulombic electrostatic interaction of $N$ atoms with point charges $\left\{q_{1}, \ldots, q_{N}\right\}$ (the non-SI unit is used, for simplicity) and positions $\left(\mathbf{r}_{1}, \ldots, \mathbf{r}_{N}\right)$ is

$E\left(\mathbf{r}_{1}, \ldots, \mathbf{r}_{N}\right) \equiv \frac{1}{2} \sum_{i=1}^{N} \sum_{j} \frac{q_{i} q_{j}}{r_{i j}}$

where $r_{i j} \equiv\left\|\mathbf{r}_{i j}\right\| \equiv\left\|\mathbf{r}_{i}-\mathbf{r}_{j}\right\|$ is the distance between atoms $i$ and $j$. The manner of summation with respect to $j$ depends on the boundary conditions.

Until recently, many of these simulations were performed by using lattice sum (LS) methods such as the Ewald method or its variants, with the assumption of the periodic boundary condition (PBC). At the beginning of these simulation studies, the cutoff truncation method, which is much simpler than the LS method, was frequently used. This is because the monotonic decreasing feature of the Coulombic potential function with increasing $r_{i j}$ allows the truncation of the interaction (Nicolas et al. 1979; Brooks et al. 1985). However, artifacts of the cutoff method have been pointed out in a number of studies (Patra et al. 2004; Saito 1994). In contrast, it has been mentioned that there are fewer artifacts when applying the PBC used in biophysical system simulations, and thus the PBC would be acceptable for such simulations (de Souza and Ornstein 1997; Buştuğ et al. 2006). Furthermore, a computationally inefficient feature of the LS method has been eliminated, using, e.g., a mesh-based approach. For these reasons, the LS method has been utilized as a standard tool.

However, quite recently, it has been understood that the artifacts of the cutoff method can be sufficiently minimized if a suitable device is added. Such devices take into account specific features, including a systemenvironment interaction, electrostatic neutrality, and a symmetry of the system, which lead to modifications of the bare Coulomb potential function. Namely, in such a cutoff-based (CB) method, interactions are defined by 
a certain pairwise function of $r_{i j}$ within a predetermined cutoff length, and the energy is represented by a finite sum of the pair function and often includes configurationirrelevant terms. In principle, they are irrelevant to boundary conditions. In contrast, artifacts in applying the $\mathrm{PBC}$ to intrinsically non-periodic systems, as well as the problems in the Ewald method and its variants, have been reconsidered. In fact, many biological systems are not intrinsically periodic and have imperfect mathematical periodicity except for certain ideal crystal states.

The CB method is simple and capable, enhancing its straightforward implementation to high-performance computational architectures, including highly parallel protocols and special purpose architectures (Kikugawa et al. 2009). Once the problems of the artifact and the accuracy are solved, the $\mathrm{CB}$ method could be more widely used because of its simplicity omitting the long range part of the interactions (Kikugawa et al. 2009; Yonezawa et al. 2011) and the irrelevance to boundary conditions.

A basic criticism of the conventional cutoff method is that an interaction truncation at only about 10-20 ̊̊ regarding the slowly decaying Coulombic potential is nothing but a complete artifact. This criticism applies to a system where the particles are spread in a broad area in a vacuum. However, this does not apply in the vivo environment, since many molecules and ions crowd over individual particles. Each positively or negatively charged particle assembles in such a way that the electrostatic interactions cancel each other well, unless very high energy phenomena suddenly occur. This feature should be the same for condensed ionic systems (Clarke et al. 1986). Thus, actual interactions in biological systems are essentially screened, as compared with the bare Coulombic form $1 / r$. In addition, considering the screened nature, we may assume that many biological processes occur through the consecution of adjacent interactions among the particles surrounding every part, rather than through distant, instantaneous interactions. These considerations provide positive motivation for employing the $\mathrm{CB}$ methods.

In fact, the $\mathrm{CB}$ methods have recently been reconsidered by many researchers, with the use of effective devices (Steinbach and Brooks 1994; Hünenberger and van Gunsteren 1998; Wolf et al. 1999; Yakub and Ronchi 2003; Fukuda et al. 2011). In this review, we discuss these efforts and the relationships among individual methods.

\section{PBC and the Ewald method}

In the 3-dimensional PBCs, the Coulombic energy of $N$ charges belonging in an MD cubic unit cell with the cell length $L$ is considered to be
$E_{\mathrm{PBC}}\left(\mathbf{r}_{1}, \ldots, \mathbf{r}_{N}\right)=\frac{1}{2} \sum_{\mathbf{n} \in \mathbb{Z}^{3}}^{\prime} \sum_{i=1}^{N} \sum_{j=1}^{N} \frac{q_{i} q_{j}}{\left\|\mathbf{r}_{i j}+L \mathbf{n}\right\|}$.

Here, $\mathbf{n}=\left(n_{1}, n_{2}, n_{3}\right)$ is a lattice point represented by three integers, and the prime on the summation indicates the omission of the $i=j$ term when $\mathbf{n}=(0,0,0)$. Throughout this review we assume total charge neutrality,

$$
\sum_{i=1}^{N} q_{i}=0
$$

which is critical to defining finite energy. Due to the slow decay of the function, in general, the summation (2) is conditionally convergent, and thus the value is completely dependent on the ordering of the summation. In other words, we should define the answer by choosing the ordering. This fact is in total contrast to the absolutely summable summation, where any order gives unique value and thus any (correct) summation method can be used, in principle.

In the periodic system, the Ewald method (Ewald 1921) has been used as the standard. According to de Leeuw et al. (1980), the Ewald energy (except for the dipole term) is interpreted to be a value of the sum, Eq. (2), obtained by a spherical-shell ordering with respect to the copies of the unit cell (i.e., image cells). Namely, the interactions from image cells that are closer to the unit cell in view of the 2-norm [viz., $\|\mathbf{n}\| \equiv\|\mathbf{n}\|_{2} \equiv\left[n_{1}^{2}+n_{2}^{2}+n_{3}^{2}\right]^{1 / 2}$ ] are counted in a preceding way. Although the counts of the interactions from certain charges that are closer to the unit cell are postponed, the above counting seems to be physically natural (e.g., as compared with counting via the cubic-shell ordering, for which another norm $\|\mathbf{n}\|_{\infty} \equiv \max \left\{\left|n_{1}\right|,\left|n_{2}\right|,\left|n_{3}\right|\right\}$ is used instead of the 2-norm). This may be the basis for justifying the definition that the Ewald sum is the answer. To effectively handle such an ordering, de Leeuw et al. employed a convergence factor, which adapts well to the ordering, in the original sum, thus yielding a weighted sum in the sphericalshell ordering. In addition, the convergence factor, which contains a parameter, has mathematically good properties that lead to absolute summability and uniform convergence with respect to the parameter.

The Ewald energy traces the properties of the energy, Eq. (2). First, it depends on the cell length $L$ : In practice, the Fourier term is influenced so it is smaller for larger values of $L$. While the Ewald energy is invariant under a translation of the axis, this is not the case for a rotation $O: \mathbf{r}_{i} \mapsto \mathrm{Or}_{i}(\forall i)$, where the coordinate value of each particle in the unit cell is transformed due to the rotation of the axis. Namely, the energy (of the original system) depends on such a rotation, in general. Invariance is ensured through particular rotations that map $\mathbb{Z}^{3}$ onto $\mathbb{Z}^{3}$; e.g. $\frac{\pi}{2}$-rotation around the z-axis. The dependences of the energy on the cell-size and the rotation do not necessarily correspond to physical reality. One 
attempt to recover the rotational invariance is seen in the pre-averaging method, demonstrated in the next section.

The above discussion is focused on possible artifacts generated from the definition of the period. However, once the definition is fixed, the energy upon the period is effectively calculated by the Ewald method (Sagui and Darden 1999). In addition, if the original system permits the periodic structure, then such an artifact can be ignored. However, the application of the PBC to an intrinsically non-periodic system often causes unignorable artifacts.

For an intrinsically non-periodic system such as aqueous protein solutions, the interactions from the infinite copies of the cell, imposed by the PBC, are clearly duplicated (Weber et al. 2000; Kastenholz and Hünenberger 2004), unless we can treat it as a crystal state. As the visible physical effect of such a PBC artifact, enforced stabilization has been observed, e.g., through lower root mean square deviations. Excessively stable alpha-helical structures for explicitly solvated polypeptides (Beck et al. 2005; Lins and Röthlisberger 2006) as well as an entrapment around a non-helical structure in a $>20$-ns simulation (Lins and Röthlisberger 2006) were found. Erratic phenomena for strain were also observed in nanowires with free surfaces (Gdoutos et al. 2010).

Extensive use of the PBC may be followed by early success in treating an isotropic bulk system. This is because the PBC allows us to avoid the creation of an interface, which often causes significant artifacts, and to mimic the bulk state. However, note that, even in a bulk system, macroscopic fluctuations and wave transport are not necessarily periodic phenomena, given the periods. Although some aspects of these artifacts may be sufficiently reduced by careful consideration of the simulation conditions, such as the cell size, dielectric constant, charge distribution, and the sampling duration, the issues are still under discussion (Hünenberger and McCammon 1999; Monticelli et al. 2006).

Finally, we note there are alternative approaches such as considering the Poisson equation in the PBC. Several methods, including those to attain a fast convergence, have been intensively developed (Tyagi 2005; see also the references therein).

\section{CB methods}

We first discuss general issues, including a truncation mode, function smoothing technique, and artifacts we should consider in cutoff approaches. Second, as a specific issue, individual $\mathrm{CB}$ methods are discussed, mainly for recently developed ones. Here, we address the reaction field (RF) method, the pre-averaging (PA) method, the Wolf method, and the zero-dipole (ZD) summation method.

To specifically perform a cutoff, as well as the pair potential function itself, a cutoff truncation mode (i.e. how to truncate the interactions) should be fixed. This is not trivial, because the choice affects the simulation results and often causes significant artifacts. In the atom-based cutoff (AC) mode, for atom $i$, the contribution from atom $j$ such that $r_{i j}>r_{c}$ is simply ignored. In the group-based cutoff (GC) mode, all atom-atom interactions between any two molecules should be on or off, according to a certain "marker" being inside or outside the cutoff sphere, respectively. Such a marker is usually chosen to be a certain center of the molecule (Baumketner 2009; Chipot et al. 1994; Neumann 1985; Schreiber and Steinhauser 1992a; van der Spoel et al. 1998), a certain atom (Alper and Levy 1989; Leach 2001), or a certain distance (Fukunishi et al. 2003; Leach 2001). Some implementations consider the marker out to a distance several Angstroms beyond the cutoff distance (this is often combined with a pair-list recycling feature). Smoothed on-off is also possible (Steinbach and Brooks 1994), and a certain-defined (e.g., neutrally charged) atom group is usually used (Wohlert and Edholm 2004), instead of a whole molecule.

Biophysical system needs an aqueous environment. Although cutoff methods have been applied to water(-like) systems, the results of the dielectric property, which would be the most sensitive property to a treatment of the electrostatic interaction (Andrea et al. 1983), often involve significant artifacts. This is particularly for the distance-dependent Kirkwood factor $G_{\mathrm{K}}(r)$, which is the ensemble average of the dipole-dipole angle distribution in the sphere of radius $r$ (Steinhauser 1982; Höchtl et al. 1998). Many cutoff methods (Yonetani 2006) yielded $G_{\mathrm{K}}(r)$ with a significant hole-like structure around $r_{\mathrm{c}}$, contradicting the expected results (Mark and Nilsson 2002).

Such artifacts appear for several reasons. First, earlier simulations suffered from limited computational time. That is, the dielectric properties require a long simulation duration, typically over a ns, to yield reliable results, because of the slow convergence of the (time) ensemble average (Heinz et al. 2001; Li et al. 2007; Gereben and Pusztai 2011). Second, ad hoc procedures, such as velocity rescaling to stabilize the system, are not recommended for evaluating sensitive properties. Finally, and often critically, the discrepancies are prominent in the GC mode, rather than the AC mode. In fact, the artifacts intrinsic in many GC modes have been pointed out. The energetic and statistical jumps were discussed (Steinhauser 1982). A clear explanation by Baumketner (2009) mentions the generation of an artificial dipole layer on the cutoff sphere of each atom: the interaction unbalances by the GC mode disrupt the charge compensation near the cutoff surface (Hummer et al. 1997).

However, as many molecular simulations utilize the GC mode in cutoff methods, there are several reasons to use it: (1) a force-field is usually developed on interactions between molecules; (2) it can prevent large energy 
fluctuations near $r_{\mathrm{c}}$, which are encountered in the straight $\mathrm{AC}$, because individual inter-atom interactions are often significantly large (Steinbach and Brooks 1994; Leach 2001); (3) if both molecules are neutral, then the leading term of their interaction can be described by a dipole-dipole interaction $\left[\sim\left(r_{\mathrm{MM}}\right)^{-3}\right]$, whose enhanced screening feature conforms to justify the cutoff (Wohlert and Edholm 2004); and (4) it is preferable for the RF method (see below) to attain the assumption of charge neutrality in the cavity, when every molecule is neutral (Neumann 1985).

If these issues can be addressed using the $\mathrm{AC}$ mode with certain other devices, then we expect the AC mode will be used to avoid the artifacts in the GC mode. Potential (or force) smoothing techniques (see, e.g., Steinbach and Brooks 1994) are useful to address issues (1) and (2). In fact, the artifacts in the dielectric properties of a water system using the GC mode were reduced by using the AC mode when employing smoothing methods, involving the force-switching or the force-shifting (Mark and Nilsson 2002; van der Spoel and van Maaren 2006). In the other systems, an artifact in explicitly solvated peptide conformation in the GC mode cutoff was obtained (Schreiber and Steinhauser 1992a), but physically reasonable behavior was confirmed in the AC mode using a force-shifting method (Beck et al. 2005). An oligonucleotide in aqueous solution can be stabilized via the AC mode with smoothing methods such as the force shift, although it failed in the GC mode (Norberg and Nilsson 2000). The issues (3) and (4) will be considered in specific CB methods.

\section{The reaction field method}

During the long history of the RF method (Onsager 1936), its effectiveness and artifacts have both been pointed out (Barker and Watts 1973; Steinhauser 1982; Neumann 1983; Hünenberger and van Gunsteren 1998; Essex 1998; Hansson et al. 2002; Gargallo et al. 2003; Robertson et al. 2008; Schulz et al. 2009; Míguez et al. 2010). The method can be viewed as a modification of the simple truncation method. It takes into account the interactions between each atom (or molecule) and the environment outside its cutoff sphere. Specifically, we consider a "cavity" of each molecule $a$ (the "cavity" resembles a cutoff sphere with radius $r_{\mathrm{c}}$ for each atom; see assumption (ii) below), with the region outside the cavity assumed to be a dielectric continuum, with dielectric constant $\varepsilon_{\mathrm{RF}}$, polarized by reacting with the molecules inside the cavity. The polarization generates an electric field (reaction field) $E_{a}$ represented by

$E_{a} \equiv \frac{2\left(\varepsilon_{\mathrm{RF}}-1\right)}{2 \varepsilon_{\mathrm{RF}}+1} \frac{1}{r_{\mathrm{c}}^{3}} \sum_{b} \mu_{b}$,

where the summation is over all molecules in the cavity of molecule $a$ and $\mu_{b} \equiv \sum_{i \in \mathrm{Mol}_{b}} q_{i} \mathbf{r}_{i}$ is the dipole of molecule $b$ (Fröhlich 1958; Allen and Tildesley 1987). Assume that (i) the total charge in each cavity equals zero, and (ii) each molecule $a$ is sufficiently small such that the set of atoms in the cavity of molecule $a$ equals the atoms in the cutoff sphere of atom $i$, for every $i$ of molecule $a$. The RF energy of the system of $M$ molecules is thus shown to be

$$
\begin{aligned}
E^{\mathrm{RF}}\left(\mathbf{r}_{1}, \ldots, \mathbf{r}_{N}\right) & \equiv-\frac{1}{2} \sum_{a=1}^{M}\left(\mu_{a} \mid E_{a}\right) \\
& =\frac{1}{2} \sum_{i=1}^{N} \sum_{\substack{j(\neq i) \\
r_{i j}<r_{\mathrm{c}}}} q_{i} q_{j} \frac{\varepsilon_{\mathrm{RF}}-1}{2 \varepsilon_{\mathrm{RF}}+1} \frac{r_{i j}^{2}}{r_{\mathrm{c}}^{3}} .
\end{aligned}
$$

Hence, the total energy is

$$
\begin{gathered}
E_{\mathrm{RF}}\left(\mathbf{r}_{1}, \ldots, \mathbf{r}_{N}\right) \equiv \frac{1}{2} \sum_{i=1}^{N} \sum_{\substack{j(\neq i) \\
r_{i j}<r_{\mathrm{c}}}} \frac{q_{i} q_{j}}{r_{i j}}+E^{\mathrm{RF}}\left(\mathbf{r}_{1}, \ldots, \mathbf{r}_{N}\right) \\
=\frac{1}{2} \sum_{i=1}^{N} \sum_{\substack{j(\neq i) \\
r_{i j}<r_{\mathrm{c}}}} q_{i} q_{j} V_{\mathrm{RF}}\left(r_{i j}\right),
\end{gathered}
$$

with

$V_{\mathrm{RF}}(r) \equiv \frac{1}{r}\left\{1+\frac{\varepsilon_{\mathrm{RF}}-1}{2 \varepsilon_{\mathrm{RF}}+1}\left(\frac{r}{r_{\mathrm{c}}}\right)^{3}\right\}$.

Considering its application to MD, however, the nonsmoothness of the energy causes problems, such as the energy non-conservation in NVE simulations. Although the physical basis is rather unclear, the potential-shifted method may serve as a remedy; viz.,

$V_{\mathrm{RF}}(r)-V_{\mathrm{RF}}\left(r_{\mathrm{c}}\right)$

is used instead of $V_{\mathrm{RF}}(r)$ for $r<r_{\mathrm{c}}$, and 0 is set for $r \geq r_{\mathrm{c}}$. The continuity of the potential function at $r=r_{\mathrm{c}}$ is thus gained, but the force function, defined by $F_{\mathrm{RF}}(r) \equiv-D V_{\mathrm{RF}}(r)$ for $r<$ $r_{\mathrm{c}}$ and $F_{\mathrm{RF}}(r) \equiv 0$ for $r \geq r_{\mathrm{c}}$, does not share in the benefit. In fact, $F_{\mathrm{RF}}$ is continuous at $r_{\mathrm{c}}$ only in the limit of $\varepsilon_{\mathrm{RF}} \rightarrow \infty$. Of course, we can treat Eq. (5) as a molecule-molecule interaction, instead of an atom-atom (site-site) interaction. In any case, the energetic non-smoothness is a significant problem in the MD simulation. In fact, the atomic velocities were rescaled at every certain timing, to prevent energy or temperature drift (Neumann 1985; Alper and Levy 1989). Although several smoothing procedures are available, an appropriate correction to the total energy, to address the potential-energy deformation, should generally be made.

Here, we discuss the remaining issues addressed in the GC mode requirements. As for issue (4), note that combination of the GC mode with the neutrality of each molecule 
is a sufficient, but not necessary, condition for assumption (i). Furthermore, other theoretical derivations or interpretations may not need such assumptions. In fact, the derivation of RF energy can be achieved without reference to the dipolar interactions (Tironi et al. 1995). Several methods, utilizing such as the Poisson equation, and extensions have been demonstrated (Hünenberger and van Gunsteren 1998; Perram and Smith 1987; Nakamura 1996). In addition, in the ZD summation method, described later, the RF method in the case of $\varepsilon_{\mathrm{RF}} \rightarrow \infty$, which is the most suitable case in view of the forcefunction continuity as stated, naturally arises, and issues (3) and (4) are addressed with the AC mode.

The RF method has also been applied to aqueous biosystems, and some of the properties were well reproduced. However, it still often generates significant artifact in the distance-dependent Kirkwood factor (Neumann 1985; Belhadj et al. 1991). As stated, also in the RF method, the artifacts in the GC mode (Neumann 1986; Alper and Levy 1989) were reduced by using the $\mathrm{AC}$ mode (Hünenberger and van Gunsteren 1998; Schulz et al. 2009). To reduce artifacts in the RF method, a large dielectric constant $\varepsilon_{\mathrm{RF}}$ may be used. In fact, the deviated structure in $G_{\mathrm{K}}(r)$ in water system is reduced as $\varepsilon_{\mathrm{RF}}$ increases (van der Spoel and van Maaren 2006). Schulz et al. (2009) obtained good results in the case of $\varepsilon_{\mathrm{RF}} \equiv \infty$. The infinite value has also been used (Neumann 1986; Essex 1998; Míguez et al. 2010; Schreiber and Steinhauser 1992b). For the water system, the potential curves with $\varepsilon_{\mathrm{RF}} \sim 80$ and $\varepsilon_{\mathrm{RF}} \equiv \infty$ a re similar (Fig. 1), and so the approximation of $\varepsilon_{\mathrm{RF}} \equiv \infty$ may be more suitable. Note that the very low dielectric constant (around 3) for proteins is derived from dried, powdered protein data. The effective value for real solvated proteins is much closer to that of water (Beck

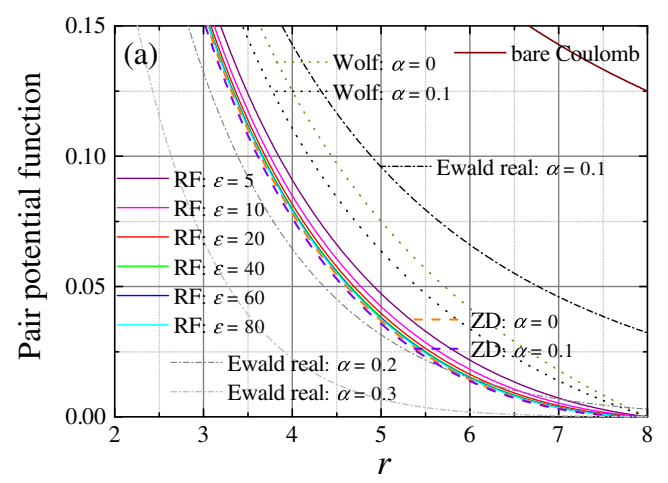

Fig. 1 Pair potential functions for $\mathrm{CB}$ methods with the cutoff length of $r_{\mathrm{c}}=8$. For the RF method, Eq. (8) is shown with the dielectric parameter $\varepsilon \equiv \varepsilon_{\mathrm{RF}}$ (curves over $\varepsilon=40$ are indistinguishable). Functions are shown for the Wolf method, $V(r)-V\left(r_{\mathrm{c}}\right)$ in Eq. (11); the ZD summation method, $u(r)-u\left(r_{\mathrm{c}}\right)$ in Eq. (15). For comparison, Eq. (12), the real part of the Ewald method, and the bare Coulomb, 1/r, are shown et al. 2005), and is 10 to 50 , depending on the pair separation distance.

There may be several other limitations in the RF method. The physically unnatural assumption of the instantaneous response by the RF in its derivation has been discussed (van Gunsteren et al. 1978). Homogeneity of the system may be required to represent the environment of each particle as a constant dielectric. However, the RF method was shown to be effective in evaluating an inhomogeneous system. For example, the interfacial properties of different water models were well described (Míguez et al. 2010), and a stable simulation using the AC mode was conducted in a solvated DNA system (while the GC mode failed; Ni and Baumketner 2011).

\section{The pre-averaging method}

The pre-averaging procedure was introduced by Yakub and Ronchi (2003), to remove the artificial cubic symmetry in the LS method and recover the rotational invariance. Their energy formula was obtained using the Ewald summation expansion and by averaging the quantities in the expansion over spherical angular coordinates. This corresponds to a situation treating a uniformly distributed system. The energy formula is

$$
\begin{aligned}
E_{\mathrm{PA}}\left(\mathbf{r}_{1}, \ldots, \mathbf{r}_{N}\right) & \equiv \frac{1}{2} \sum_{i=1}^{N} \sum_{\substack{j(\neq i) \\
r_{i j}<r_{\mathrm{m}}}} q_{i} q_{j} \frac{1}{r_{i j}}\left\{1+\frac{1}{2} \frac{r_{i j}}{r_{\mathrm{m}}}\left[\left(\frac{r_{i j}}{r_{\mathrm{m}}}\right)^{2}-3\right]\right\} \\
& -\frac{3}{4 r_{\mathrm{m}}} \sum_{i=1}^{N} q_{i}^{2},
\end{aligned}
$$

where $r_{\mathrm{m}}$ is the radius of the volume-equivalent sphere of the MD cubic cell with edge $L$ :

$\frac{4}{3} \pi r_{\mathrm{m}}^{3}=L^{3}$.

The PA method has been very successfully applied (Yakub et al. 2007; Arima et al. 2009; Jha et al. 2010). It yields accurate energy in disordered systems, including a one-component plasma and a two-component fluid (Yakub and Ronchi 2005; Yakub 2006), and, further, in nonspherical, crystal systems (Yakub and Ronchi 2003; 2005). Moreover, Guerrero-García et al. (2011) applied the PA method to inhomogeneous systems, constituted by two fixed nanoparticles immersed in a size-asymmetric monovalent electrolyte.

However, the cutoff length, $r_{\mathrm{m}}$, intrinsic to the PA formalism, causes severe practical limitations in certain cases. Namely, since $r_{\mathrm{m}}$ is proportional to the cell size $L$ and is larger than $L / 2$, enormous computational efforts will be required to treat a large system, for example in biological systems where the size is $>\mathrm{nm}$. Reinterpretation of 
the cutoff length is possible in the ZD summation method, as described below. See also the recent work by Vernizzi et al. (2011).

\section{The Wolf method}

Here, we briefly review the Wolf method and its variants, for which the effectiveness in terms of the accuracy and computational cost have been demonstrated in many applications (Wolf et al. 1999; Demontis et al. 2001; Zahn et al. 2002; Fennell and Gezelter 2006; Avendaño and Gil-Villegas 2006; Sepliarsky et al. 2006; Ribeiro 2007; Desai 2007; Goto et al. 2007; Mahadevan and Garofalini 2007; Nagata and Mukamel 2010; Chen et al. 2010; Kuang and Gezelter 2010; Gdoutos et al. 2010; Chevrot et al. 2011; Kannam et al. 2012; Méndez and Villegas 2012).

In seeking the Madelung energies of crystal systems via the straight cutoff method, Wolf (1992) and Wolf et al. (1999) showed that the energy exhibited very slow convergence and oscillated around the exact value as the cutoff length increased, but the value was very accurate only when certain cutoff lengths, characteristic of individual crystal structures, were adopted. They found that this feature was not only due to the oscillating feature but also to the (near) achievement of the charge neutrality in the cutoff spheres with those characteristic cutoff lengths. They also found that the energy error was nearly proportional to the net charge in each sphere. These observations led to the idea of the subtracting the interactions by excess charges in the cutoff sphere, $q_{i}\left(\sum_{j, r_{i j}<r_{\mathrm{c}}} q_{j}\right) / r_{\mathrm{c}}$, from the straight pairwise sum. Here, for the excess charges, their total quantities are equal to the net charge in the sphere, and their positions are assumed to be at the surface. Hence, they led to a formula for the electrostatic energy,

$$
\begin{aligned}
E_{\text {Wolf }}\left(\mathbf{r}_{1}, \ldots, \mathbf{r}_{N}\right) & \equiv \frac{1}{2} \sum_{i=1}^{N} \sum_{\substack{j(\neq i) \\
r_{i j}<r_{\mathrm{c}}}} q_{i} q_{j}\left[V\left(r_{i j}\right)-V\left(r_{\mathrm{c}}\right)\right] \\
& -\left[\frac{V\left(r_{\mathrm{c}}\right)}{2}+\frac{\alpha}{\sqrt{\pi}}\right] \sum_{i=1}^{N} q_{i}^{2} .
\end{aligned}
$$

Here, instead of $1 / r$, the damped function,

$$
V(r) \equiv \frac{\operatorname{erfc}(\alpha r)}{r},
$$

was introduced, since it was very effective to achieve a fast convergence. The last term was derived by an approximation, $\frac{1}{2} \sum_{i=1}^{N} \sum_{j(\neq i)} q_{i} q_{j}\left[1 / r_{i j}-V\left(r_{i j}\right)\right] \sim-(\alpha / \sqrt{\pi}) \sum_{i=1}^{N} q_{i}^{2}$, for a small damping factor $\alpha(\geq 0)$ (Wolf et al. 1999; but see Angoshtari and Yavari 2011).

Equation (11) can be viewed as considering the contributions from image charges; viz., for every atom in the cutoff sphere around $i$, the opposite signed image-charge exists on the surface and interacts with (only) $i$. Since the straightforward differentiation of Eq. (11) leads to the discontinuous scheme at $r=r_{\mathrm{c}}$, further consideration is needed to define an atomic force for MD. Analogous to the effective potential $V(r)-V\left(r_{\mathrm{c}}\right)$ reflecting the view of the image charges, one might suppose an effective force function, $F_{\text {Wolf }}(r) \equiv-\left[D V(r)-D V\left(r_{\mathrm{c}}\right)\right]$. Although this "force" satisfies the continuity, unfortunately it is not compatible with the energy function Eq. (11). These problems arise from a straightforward interpretation of the image-charge picture.

The second view of Eq. (11) can be obtained when one considers its first term to be the shifted-potential method for $V$. In developing such a potential-deformation picture (Zahn et al. 2002; Fennell and Gezelter 2006), the shifted-force method for the force $-D V$ was proposed. This means that the force function is given by $F_{\text {Wolf }}$, and that the corresponding potential energy is constructed by the integration. Many physical properties can be traced in these approaches. However, note that the physical basis would be unclear, and the treatment of the self image term (2nd term in Eq. (11)) becomes ambiguous.

The third view of Eq. (11) is that the energy can be obtained from a suitably-defined neutralized summation. That is, it is derived from the assertion that the interaction contribution should be counted in a neutralized subset $L^{i}$ (including $i$ ), whose existence is assumed and characterized where: (a) any particle in $L^{i}$ is inside the cutoff sphere; (b) the total charge in $L^{i}$ is zero; and (c) a particle not belonging to $L^{i}$ but inside the sphere is located close to the cutoff surface. Based on this physically clear view, the force-switching Wolf method (Fukuda et al. 2008) established an approximation to the neutralized summations, and the energy becomes

$$
\begin{aligned}
E_{\mathrm{FSw}-W o l f}\left(\mathbf{r}_{1}, \ldots, \mathbf{r}_{N}\right) & \equiv \frac{1}{2} \sum_{i=1}^{N} \sum_{\substack{j \neq i) \\
r_{i j}<r_{\mathrm{c}}}} q_{i} q_{j}\left[\widehat{V}\left(r_{i j}\right)-\widehat{V}\left(r_{\mathrm{c}}\right)\right] \\
& -\left[\frac{\widehat{V}\left(r_{\mathrm{c}}\right)}{2}+\frac{\alpha}{\sqrt{\pi}}\right] \sum_{i=1}^{N} q_{i}^{2},
\end{aligned}
$$

where

$$
\widehat{V}(r) \equiv\left\{\begin{array}{ccc}
V(r) & \text { for } & 0<r<r_{1} \\
V^{*}(r)+V\left(r_{1}\right)-V^{*}\left(r_{1}\right) & \text { for } & r_{1} \leq r \leq r_{\mathrm{c}} \\
V\left(r_{1}\right)-V^{*}\left(r_{1}\right) & \text { for } & r_{\mathrm{c}}<r<\infty
\end{array}\right.
$$

with $r_{1}$ being the switching length and $V^{*}$ a suitable switching function. This provides consistent potential and force functions, and smoothness for safely conducting MD simulations. This is simply the Wolf formula [Eq. (11)] with $\widehat{V}$ instead of $V$ (Eq. (11) is recovered as $r_{1} \rightarrow r_{\mathrm{c}}$ ). It was applied to calculate the free energies of an alanine dipeptide in explicit water, and reliable results were obtained (Yonezawa et al. 2011). 


\section{The ZD summation method}

In developing the neutralizing principle, the ZD summation method (Fukuda et al. 2011) provides the energy derived by counting the interactions for a neutralized subset regarding the dipoles as well as the charges. Thus, the ZD summation method can be viewed as an extension of the Wolf method. It effectively avoids the nonzero-dipole and nonzero-charge state artificially generated in the simple cutoff scheme. Its physical basis is clear, and the axiomatic approach ensures there is no confusion in defining its energy,

$$
\begin{aligned}
E_{\mathrm{ZD}}\left(\mathbf{r}_{1}, \ldots, \mathbf{r}_{N}\right) & =\frac{1}{2} \sum_{i=1}^{N} \sum_{\substack{j(\neq i) \\
r_{i j}<r_{\mathrm{c}}}} q_{i} q_{j}\left[u\left(r_{i j}\right)-u\left(r_{\mathrm{c}}\right)\right] \\
& -\left[\frac{u\left(r_{\mathrm{c}}\right)}{2}+\frac{\alpha}{\sqrt{\pi}}\right] \sum_{i=1}^{N} q_{i}^{2},
\end{aligned}
$$

where

$u(r) \equiv V(r)-\frac{1}{2} \frac{D V\left(r_{\mathrm{c}}\right)}{r_{\mathrm{c}}} r^{2}$.

Equation (15) is simple enough, and also takes the form of Eq. (11) using $u$ instead of $V$. In addition to the information obtained in the zero charge scheme by the Wolf approach such that the excess charge is on the cutoff sphere, the zero dipole condition adds information about the states, which helps to improve the accuracy. Furthermore, this scheme accepts the AC mode well, and the above-mentioned four issues regarding the GC mode are sufficiently addressed. In fact, the pair function smoothly tends to zero at $r_{\mathrm{c}}$, and the remaining issues (3) and (4) can be cleared by including the damping factor and considering the neutralized condition through the whole cutoff sphere, rather than each molecule.

\section{Relationship to other CB methods}

Thus far, we have discussed the RF, PA, and Wolf methods. These methods differ in their concepts, derivations, and the energy formulae themselves. Surprisingly, we see that they are related to each other by considering the connection of the individuals to the $\mathrm{ZD}$ summation method, through special limits of the parameters.

As well as the fact that the ZD summation method is an ideaistic extension of the Wolf method, function $u$ [Eq. (16)] tends to function $V$ [Eq. (12)] as $r_{\mathrm{c}} \rightarrow \infty$, and the difference of the pair potentials and that of the constant terms between the two methods approach 0 as $r_{\mathrm{c}} \rightarrow \infty$ (they also approach 0 as $\alpha \rightarrow \infty)$.
For the PA method, note that its energy, Eq. (9), can be rewritten as

$$
\begin{aligned}
E_{\mathrm{PA}}\left(\mathbf{r}_{1}, \ldots, \mathbf{r}_{N}\right) & =\frac{1}{2} \sum_{i=1}^{N} \sum_{\substack{j(\neq i) \\
r_{i j}<r_{\mathrm{c}}}} q_{i} q_{j}\left[V_{\mathrm{PA}}\left(r_{i j}\right)-V_{\mathrm{PA}}\left(r_{\mathrm{m}}\right)\right] \\
& -\frac{V_{\mathrm{PA}}\left(r_{\mathrm{m}}\right)}{2} \sum_{i=1}^{N} q_{i}^{2}
\end{aligned}
$$

where $V_{\mathrm{PA}}(r) \equiv(1 / r)\left\{1+\left(\frac{1}{2}\right)\left(r / r_{\mathrm{m}}\right)^{3}\right\}$. We see that the energy of the ZD summation, Eq. (15), gives Eq. (17) when we set $\alpha=0$ and $r_{\mathrm{c}}=r_{\mathrm{m}}$, noting that the potential function $u$ then corresponds to $V_{\mathrm{PA}}$. This fact enhances the free use of the cutoff length, which is not limited to $r_{\mathrm{m}}$, in the PA method, and might explain why the PA method yields "surprisingly positive" results in anisotropic systems (Yakub and Ronchi 2003).

In the RF method, a pairwise sum is conducted by $V_{\mathrm{RF}}$ [Eq. (7)] (Neumann 1985) or by the shifted form $V_{\mathrm{RF}}{ }^{-}$ $V_{\mathrm{RF}}\left(r_{\mathrm{c}}\right)$ [Eq. (8)] (van der Spoel and van Maaren 2006; Baumketner 2009). Note that $V_{\mathrm{RF}}$ with $\varepsilon_{\mathrm{RF}} \rightarrow \infty$ corresponds to $u$ with $\alpha=0$. In other words, if we propose a modified RF summation by

$$
\begin{gathered}
E_{\mathrm{MRF}}\left(\mathbf{r}_{1}, \ldots, \mathbf{r}_{N}\right) \equiv \frac{1}{2} \sum_{i=1}^{N} \sum_{\substack{j(\neq i) \\
r_{i j}<r_{\mathrm{c}}}} q_{i} q_{j}\left[V_{\mathrm{RF}}\left(r_{i j}\right)-V_{\mathrm{RF}}\left(r_{\mathrm{c}}\right)\right] \\
-\frac{V_{\mathrm{RF}}\left(r_{\mathrm{c}}\right)}{2} \sum_{i=1}^{N} q_{i}^{2}
\end{gathered}
$$

then its limit as $\varepsilon_{\mathrm{RF}} \rightarrow \infty$ equals the ZD summation energy (15) with $\alpha=0$. The difference between the RF energy and the modified one is the "self energy," viz. the last term in Eq. (18).

\section{Other methods}

Other promising $\mathrm{CB}$ approaches exist. The ZD summation method can be extended to accommodate higher multipoles. The force-matching method (Ercolessi and Adams 1994; Shi et al. 2008), the isotropic periodic summation (Wu and Brooks 2005; 2008), and the screening scheme using the Yukawa potential (Carré et al. 2007) are highly effective. Important new non-Ewald electrostatics methods have been developed, including local molecular field theory (Chen and Weeks 2006), a fast multipole method combined with a reaction field (Mathias et al. 2003), the lattice-sumemulated reaction-field method (Heinz and Hünenberger 2005), an image-charge reaction field method (Lin et al. 
2009, 2011), and a model of electrostatic and liquidstructure forces (Hassan 2007).

\section{Conclusion}

After reviewing intrinsic aspects of the $\mathrm{PBC}$ with the LS method, we mainly considered the $\mathrm{CB}$ methods including the conventional RF method and the recently-developed PA, Wolf, and ZD summation methods.

Although the PBC with the LS method has been most frequently applied to biomolecular systems, they are still far from reality, and some artifacts have been recognized. The CB method could provide a promising solution, and, because of its simple features, it could satisfy the demands of high-performance computational architectures. Investigation of the RF method using the AC mode should be continued. Pursuit of symmetry of the system, as considered in the PA method, will be useful in particular for reconsidering the boundary conditions. Although more considerations may be required, the relationships between the $\mathrm{CB}$ methods suggest new interpretations and extensive applications. The "interactions" among the individual CB methods, even with the LS method, should mature the algorithm for calculating electrostatic interactions.

Acknowledgement This research was supported by Research and Development of the Next-Generation Integrated Simulation of Living Matter, a part of the Development and Use of the Next-Generation Supercomputer Project of the Ministry of Education, Culture, Sports, Science and Technology of Japan.

\section{Conflict of interest None.}

Open Access This article is distributed under the terms of the Creative Commons Attribution License which permits any use, distribution, and reproduction in any medium, provided the original author(s) and the source are credited.

\section{References}

Allen MP, Tildesley DJ (1987) Computer simulations of liquids. Oxford University Press, New York

Alper HE, Levy RM (1989) Computer simulations of the dielectric properties of water: studies of the simple point charge and transferrable intermolecular potential models. J Chem Phys 91:12421251

Andrea TA, Swope WC, Andersen HC (1983) The role of long ranged forces in determining the structure and properties of liquid water. J Chem Phys 79:4576-4584

Angoshtari A, Yavari A (2011) Convergence analysis of the Wolf method for coulombic interactions. Phys Lett A 375:1281-1285

Arima T, Idemitsu K, Inagaki Y, Tsujita Y, Kinoshita M, Yakub E (2009) Evaluation of melting point of UO2 by molecular dynamics simulation. J Nucl Mater 389:149-154
Avendaño C, Gil-Villegas A (2006) Monte Carlo simulations of primitive models for ionic systems using the Wolf method. Mol Phys 104:1475-1486

Barker JA, Watts RO (1973) Monte Carlo studies of the dielectric properties of water-like models. Mol Phys 26:789-792

Baumketner A (2009) Removing systematic errors in interionic potentials of mean force computed in molecular simulations using reaction-field-based electrostatics. J Chem Phys 130:104106

Beck DA, Armen RS, Daggett V (2005) Cutoff size need not strongly influence molecular dynamics results for solvated polypeptides. Biochemistry 44:609-616

Belhadj M, Alper HE, Levy RM (1991) Molecular dynamics simulations of water with Ewald summation for the long range electrostatic interactions. Chem Phys Lett 179:13-20

Brooks CL, Pettitt BM, Karplus M (1985) Structural and energetic effects of truncating long ranged interactions in ionic and polar fluids. J Chem Phys 83:5897-5908

Buştuğ T, Patra SM, Kuyucak S (2006) Finite system and periodicity effects in free energy simulations of membrane proteins. Chem Phys Lett 425:320-323

Carré A, Berthier L, Horbach J, Ispas S, Kob W (2007) Amorphous silica modeled with truncated and screened Coulomb interactions: a molecular dynamics simulation study. J Chem Phys 127:114512

Chen YG, Weeks JD (2006) Local molecular field theory for effective attractions between like charged objects in systems with strong Coulomb interactions. Proc Natl Acad Sci USA 103:7560-7565

Chen DL, Stern AC, Space B, Johnson JK (2010) Atomic charges derived from electrostatic potentials for molecular and periodic systems. J Phys Chem A 114:10225-10233

Chevrot G, Calligari P, Hinsen K, Kneller GR (2011) Least constraint approach to the extraction of internal motions from molecular dynamics trajectories of flexible macromolecules. J Chem Phys 135:084110

Chipot C, Millot C, Maigret B, Kollman PA (1994) Molecular dynamics free energy simulations: influence of the truncation of longrange nonbonded electrostatic interactions on free energy calculations of polar molecules. J Chem Phys 101:7953-7962

Clarke JHR, Smith W, Woodcock LV (1986) Short range effective potentials for ionic fluids. J Chem Phys 84:2290-2294

de Leeuw SW, Perram JW, Smith ER (1980) Simulation of electrostatic systems in periodic boundary conditions. I. Lattice sums and dielectric constants. Proc R Soc Lond A 373:27-56

de Souza ON, Ornstein R (1997) Effect of periodic box size on aqueous molecular dynamics simulation of a DNA dodecamer with particle-mesh Ewald method. Biophys J 72:2395-2397

Demontis P, Spanu S, Suffritti GB (2001) Application of the Wolf method for the evaluation of Coulombic interactions to complex condensed matter systems: aluminosilicates and water. J Chem Phys 114:7980-7988

Desai TG (2007) Molecular dynamics study of screening at ionic surfaces. J Chem Phys 127:154707

Ercolessi F, Adams JB (1994) Interatomic potentials from first-principles calculations: the force-matching method. Europhys Lett 26:583-588

Essex JW (1998) The application of the reaction-field method to the calculation of dielectric constants. Mol Sim 20:159-178

Ewald PP (1921) Die Berechnung optischer und elektrostatischer Gitterpotentiale. Ann Phys (Leipzig) 64:253-287

Fennell CJ, Gezelter JD (2006) Is the Ewald summation still necessary? Pairwise alternatives to the accepted standard for long-range electrostatics. J Chem Phys 124:234104

Fröhlich H (1958) Theory of dielectrics. Clarendon, Oxford

Fukuda I, Yonezawa Y, Nakamura H (2008) Consistent molecular dynamics scheme applying the Wolf summation for calculating electrostatic interaction of particles. J Phys Soc Jpn 77:114301 
Fukuda I, Yonezawa Y, Nakamura H (2011) Molecular dynamics scheme for precise estimation of electrostatic interaction via zero-dipole summation principle. J Chem Phys 134:164107

Fukunishi Y, Mikami Y, Nakamura H (2003) The filling potential method: a method for estimating the free energy surface for protein-ligand docking. J Phys Chem B 107:13201-13210

Gargallo R, Hünenberger PH, Avilés FX, Oliva B (2003) Molecular dynamics simulation of highly charged proteins: comparison of the particle-particle particle-mesh and reaction field methods for the calculation of electrostatic interactions. Protein Sci 12:21612172

Gdoutos EE, Agrawal R, Espinosa HD (2010) Comparison of the Ewald and Wolf methods for modeling electrostatic interactions in nanowires. Int J Numer Meth Eng 84:1541-1551

Gereben O, Pusztai L (2011) On the accurate calculation of the dielectric constant from molecular dynamics simulations: the case of SPC/E and SWM4-DP water. Chem Phys Lett 507:80-83

Goto M, Takezoe H, Ishikawa K (2007) Carrier transport simulation of anomalous temperature dependence in nematic liquid crystals. Phys Rev E 76:040701(R)

Guerrero-García GI, Mozuelos PG, Olvera de la Cruz M (2011) Potential of mean force between identical charged nanoparticles immersed in a size-asymmetric monovalent electrolyte. J Chem Phys 135:164705-164714

Hansson T, Oostenbrink C, van Gunsteren WF (2002) Molecular dynamics simulations. Curr Opin Struct Biol 12:190-196

Hassan SA (2007) Liquid-structure forces and electrostatic modulation of biomolecular interactions in solution. J Phys Chem B 111:227241

Heinz TN, Hünenberger PH (2005) Combining the lattice-sum and reaction-field approaches for evaluating long-range electrostatic interactions in molecular simulations. J Chem Phys 123:034107

Heinz TN, van Gunsteren WF, Hünenberger PH (2001) Comparison of four methods to compute the dielectric permittivity of liquids from molecular dynamics simulations. J Chem Phys 115:11251136

Höchtl P, Boresch S, Bitomsky W, Steinhauser O (1998) Rationalization of the dielectric properties of common three-site water models in terms of their force field parameters. J Chem Phys 109:4927-4937

Hummer G, Pratt LR, García AE, Berne BJ, Rick SW (1997) Electrostatic potentials and free energies of solvation of polar and charged molecules. Phys Chem B 101:3017-3020

Hünenberger PH, McCammon JA (1999) Effect of artificial periodicity in simulations of biomolecules under Ewald boundary conditions: a continuum electrostatics study. Biophys Chem 78:69-88

Hünenberger PH, van Gunsteren WF (1998) Alternative schemes for the inclusion of a reaction-field correction into molecular dynamics simulations: influence on the simulated energetic, structural, and dielectric properties of liquid water. J Chem Phys 108:61176134

Jha PK, Sknepnek R, Guerrero-García GI, Olvera de la Cruz M (2010) A graphics processing unit implementation of coulomb interaction in molecular dynamics. J Chem Theory Comput 6:30583065

Kannam SK, Todd BD, Hansen JS, Daivis PJ (2012) Slip length of water on graphene: limitations of non-equilibrium molecular dynamics simulations. J Chem Phys 136:024705

Kastenholz M, Hünenberger PH (2004) Influence of artificial periodicity and ionic strength in molecular dynamics simulations of charged biomolecules employing lattice-sum methods. J Phys Chem B 108:774-788

Kikugawa G, Apostolov R, Kamiya N, Taiji M, Himeno R, Nakamura H, Yonezawa Y (2009) Application of MDGRAPE-3, a special purpose board for molecular dynamics simulations, to periodic biomolecular systems. J Comput Chem 30:110-118
Koehl P (2006) Electrostatics calculations: latest methodological advances. Curr Opin Struct Biol 16:142-151

Kuang S, Gezelter JD (2010) A gentler approach to RNEMD: nonisotropic velocity scaling for computing thermal conductivity and shear viscosity. J Chem Phys 133:164101

Leach AR (2001) Molecular modelling: principles and applications, 2nd ed. Prentice Hall, Englewood Cliffs

Li J, Zhou Z, Sadus RJ (2007) Role of nonadditive forces on the structure and properties of liquid water. J Chem Phys 127:154509

Lin Y, Baumketner A, Deng S, Xu Z, Jacobs D, Cai W (2009) An image-based reaction field method for electrostatic interactions in molecular dynamics simulations of aqueous solutions. J Chem Phys 131:154103

Lin Y, Baumketner A, Song W, Deng S, Jacobs D, Cai W (2011) Ionic solvation studied by image-charge reaction field method. J Chem Phys 134:044105

Lins RD, Röthlisberger U (2006) Influence of long-range electrostatic treatments on the folding of the N-Terminal H4 histone tail peptide. J Chem Theory Comput 2:246-250

Mahadevan TS, Garofalini SH (2007) Dissociative water potential for molecular dynamics simulations. J Phys Chem B 111:89198927

Mark P, Nilsson L (2002) Structure and dynamics of liquid water with different long-range interaction truncation and temperature control methods in molecular dynamics simulations. J Comput Chem 23:1211-1219

Mathias G, Egwolf G, Nonella M, Tavan P (2003) A fast multipole method combined with a reaction field for long-range electrostatics in molecular dynamics simulations: the effects of truncation on the properties of water. J Chem Phys 118:1084710860

Méndez PXV, Villegas AG (2012) Computer simulation of sedimentation of ionic systems using the Wolf method. J Chem Phys 136:154507

Míguez JM, González-Salgado D, Legido JL, Piñeiro MM (2010) Calculation of interfacial properties using molecular simulation with the reaction field method: results for different water models. J Chem Phys 132:184102

Monticelli L, Simões C, Belvisi L, Colombo G (2006) Assessing the influence of electrostatic schemes on molecular dynamics simulations of secondary structure forming peptides. J Phys Cond Matt 18:S329-S345

Nagata Y, Mukamel S (2010) Vibrational sum-frequency generation spectroscopy at the water/lipid interface: molecular dynamics simulation study. J Am Chem Soc 132:6434-6442

Nakamura H (1996) Roles of electrostatic interaction in proteins. Quart Rev Biophys 29:1-90

Neumann M (1983) Dipole moment fluctuation formulas in computer simulations of polar systems. Mol Phys 50:841-858

Neumann M (1985) The dielectric constant of water. Computer simulations with the MCY potential. J Chem Phys 82:5663-5672

Neumann M (1986) Dielectric relaxation in water. Computer simulations with the TIP4P potential. J Chem Phys 85:1567-1580

Ni B, Baumketner A (2011) Effect of atom- and group-based truncations on biomolecules simulated with reaction-field electrostatics. J Mol Model 17:2883-2893

Nicolas JJ, Gubbins KE, Streett WB, Tildesley DJ (1979) Equation of state for the Lennard-Jones fluid. Mol Phys 37:1429-1454

Norberg, Nilsson (2000) On the truncation of long-range electrostatic interactions in DNA. Biophys J 79:1537-1553

Onsager L (1936) Electric moments of molecules in liquids. J Am Chem Soc 58:1486-1493

Patra M, Karttunen M, Hyvönen MT, Falck E, Vattulainen I (2004) Lipid bilayers driven to a wrong lane in molecular dynamics simulations by subtle changes in long-range electrostatic interactions. J Phys Chem B 108:4485-4494 
Perram JW, Smith ER (1987) Microscopic derivation of fluctuation formulas for calculating dielectric constants by simulation. J Stat Phys 46:179-190

Reif MM, Krautler V, Kastenholz MA, Daura X, Hünenberger PH (2009) Molecular dynamics simulations of a reversibly folding $\beta$-heptapeptide in methanol: influence of the treatment of longrange electrostatic interactions. J Phys Chem B 113:3112-3128

Ribeiro MCC (2007) High frequency sound velocity in the glass former $2 \mathrm{Ca}(\mathrm{NO} 3) 2 \cdot 3 \mathrm{KNO} 3$ : molecular dynamics simulations. Phys Rev B 75:144202

Robertson A, Luttmann E, Pande VS (2008) Effects of long-range electrostatic forces on simulated protein folding kinetics. J Comput Chem 29:694-700

Sagui C, Darden TA (1999) Molecular dynamics simulations of biomolecules: long-range Electrostatic Effects. Annu Rev Biophys Biomol Struct 28:155-179

Saito M (1994) Molecular dynamics simulations of proteins in solution: artifacts caused by the cutoff approximation. J Chem Phys 101:4055-4061

Schreiber H, Steinhauser O (1992a) Cutoff size does strongly influence molecular dynamics results on solvated polypeptides. Biochemistry 31:5856-5860

Schreiber H, Steinhauser O (1992b) Taming cut-off induced artifacts in molecular dynamics studies of solvated polypeptides: the reaction field method. J Mol Biol 228:909-923

Schulz R, Lindner B, Petridis L, Smith JC (2009) Scaling of multimillion-atom biological molecular dynamics simulation on a petascale supercomputer. J Chem Theor Comput 5:2798-2808

Sepliarsky M, Stachiotti MG, Migoni RL (2006) Interface effects in ferroelectric $\mathrm{PbTiO}_{3}$ ultrathin films on a paraelectric substrate. Phys Rev Lett 96:137603

Shi Q, Liu P, Voth GA (2008) Coarse-graining in interaction space: an analytical approximation for the effective short-ranged electrostatics. J Phys Chem B 112:16230-16237

Srivastava S, Santos A, Critchley K, Kim KS, Podsiadlo P, Sun K, Lee J, Xu C, Lilly GD, Glotzer SC, Kotov NA (2010) Light-controlled self-assembly of semiconductor nanoparticles into twisted ribbons. Science 327:1355-1359

Steinbach PJ, Brooks BR (1994) New spherical-cutoff methods for long-range forces in macromolecular simulation. J Comput Chem $15: 667-683$

Steinhauser O (1982) Reaction field simulation of water. Mol Phys 45:335-348

Tironi IG, Sperb R, Smith PE, van Gunsteren WF (1995) A generalized reaction field method for molecular dynamics simulations. J Chem Phys 102:5451-5459

Tyagi S (2005) Coulomb potentials in two and three dimensions under periodic boundary conditions. J Chem Phys 122:014101 van der Spoel D, van Maaren PJ (2006) The origin of layer structure artifacts in simulations of liquid water. J Chem Theor Comput 2:1-11

van der Spoel D, van Maaren PJ, Berendsen HJC (1998) A systematic study of water models for molecular simulation: derivation of water models optimized for use with a reaction field. J Chem Phys 108:10220-10230

van Gunsteren WF, Berendsen HJC, Rullmann JAC (1978) Inclusion of reaction fields in molecular dynamics. Application to liquid water. Faraday Discuss Chem Soc 66:58-70

Vernizzi G, Guerrero-García GI, Olvera de la Cruz M (2011) Coulomb interactions in charged fluids. Phys Rev E 84:016707

Weber W, Hünenberger PH, McCammon JA (2000) Molecular dynamics simulations of a polyalanine octapeptide under Ewald boundary conditions: influence of artificial periodicity on peptide conformation. J Phys Chem B 104:3668-3675

Wohlert J, Edholm O (2004) The range and shielding of dipole-dipole interactions in phospholipid bilayers. Biophys J 87:2433-2445

Wolf $\mathrm{D}$ (1992) Reconstruction of $\mathrm{NaCl}$ surfaces from a dipolar solution to the Madelung problem. Phys Rev Lett 68:3315-3318

Wolf D, Keblinski P, Phillpot SR, Eggebrecht J (1999) Exact method for the simulation of Coulombic systems by spherically truncated, pairwise $r^{-1}$ summation. J Chem Phys 110:8254-8282

Wu X, Brooks BR (2005) Isotropic periodic sum: a method for the calculation of long-range interactions. J Chem Phys 122:044107

Wu X, Brooks BR (2008) Using the isotropic periodic sum method to calculate long-range interactions of heterogeneous systems. J Chem Phys 129:154115

Yakub E (2006) Effective computer simulation of strongly coupled Coulomb fluids. J Phys A 39:4643-4649

Yakub E, Ronchi C (2003) An efficient method for computation of long-ranged Coulomb forces in computer simulation of ionic fluids. J Chem Phys 119:11556-11560

Yakub E, Ronchi C (2005) A new method for computation of long ranged coulomb forces in computer simulation of disordered systems. J Low Temp Phys 139:633-643

Yakub E, Ronchi C, Staicu D (2007) Molecular dynamics simulation of premelting and melting phase transitions in stoichiometric uranium dioxide. J Chem Phys 127:094508

Yonetani Y (2006) Liquid water simulation: a critical examination of cutoff length. J Chem Phys 124:204501

Yonezawa Y, Fukuda I, Kamiya N, Shimoyama H, Nakamura H (2011) Free energy landscapes of alanine dipeptide in explicit water reproduced by the force-switching Wolf method. J Chem Theor Comput 7:1484-1493

Zahn D, Schilling B, Kast SM (2002) Enhancement of the Wolf damped coulomb potential: static, dynamic, and dielectric properties of liquid water from molecular simulation. J Phys Chem B 106:10725-10732 